PAPER

\title{
Adaptive Multi-Directional Max-Plus Algebra-Based Morphological Wavelet Transform
}

\author{
Takeshi Kumaki, Yuri Tanito, Tatsuki Tokunaga, Tomohiro Fujita and Takeshi Ogura \\ Department of Electronic and Computer Engineering, Ritsumeikan University, 1-1-1 Noji-Higashi, Kusatsu, Shiga \\ 525-8577, Japan \\ E-mail: \{kumaki@fc, ri005088@ed,ri0004lx@ed, tfujita@se, togura@se\}.ritsumei.ac.jp
}

\begin{abstract}
This paper presents an Adaptive Multi-directional Max-plus algebra-based Morphological wavelet Transform (AM-MMT). The AM-MMT is based on a conventional max-plus algebra-based morphological wavelet transform and utilizes several suitable sampling windows that are adaptively selected in accordance with the direction of the content in the image. Thus, this proposed method extracts directional structures smoothly to calculate nonlinear operation (maximum or minimum search) and the standard sum. To show the effectiveness of the AM-MMT, nine standard benchmark images were used to compare the AM-MMT with the conventional MMT. From the experiment, transformed-images can be made by combining the high quality parts of the images, which are processed by each sampling window. All the PSNR values of the AM-MMT are higher than those of the conventional MMT with increasing deletion bit width. Thus, the AM-MMT achieves high-quality high-compression digital images. Furthermore, the expansion into the multi-level AM-MMT operation is described, and a Level 2 (L2) implementation example is shown. The L2 AM-MMT compressed-image is up to about $82 \%$ smaller than the original image. Consequently, the AM-MMT can accomplish effective nonlinear operation-based image transformation.
\end{abstract}

Keywords: max-plus algebra, morphological wavelet transform, directional decomposition, directional reconstruction, data compression, image quality, image processing

\section{Introduction}

The use of mobile devices, such as cellular phones and smartphones, has spread due to the rapid development of semiconductor integration and embedded LSI technology. Thus, several types of mobile applications execute digital content in real-time to satisfy userrequirements. Therefore, the image coding algorithm is most widely used for several multimedia applications. Generally, for effective image coding, several transform algorithms are widely used in many parts of multimedia applications. However, some standard 2-D transforms used in image coding are often executed separately in the vertical and horizontal directions. Then, all images, such as natural scenes, people and structures, that contain various forms cannot be sufficiently supported in the vertical or horizontaldirection. In previous researches [1]-[12], several directional image coding techniques have been extracted that provide some directional information from the original image that can be developed for improving im- age quality. However, these algorithms often deal with the field of formulation of real numbers and several complex mathematical equations. These methods are thus difficult to implement with general-purpose processors, especially low-power embedded mobile processors in cellular phones and smartphones. For overcoming these problems, a max-plus algebra-based morphological wavelet transform has been focused on to have both high-quality and high-compression for fast image processing on mobile embedded processors [13]-[19]. This transform is rich in potential for effective directional image coding. In this paper, an Adaptive Multi-directional Max-plus algebra-based Morphological wavelet Transform (AM-MMT) is proposed and evaluated for improving the efficiency of creating quality images by data decomposition and reconstruction processing. The proposed method realizes a novel image transformation technique for adapting a suitable sampling window selection, which is decided in accordance with the directional features in the image. To determine the best direction for a suitable sampling 
window, the AM-MMT extracts directional structures smoothly to calculate nonlinear operation (maximum or minimum search) and the standard sum. In this transform, macro blocks, which are created by dividing the original image in a reticular pattern, can be selected from candidate sampling windows for fitting image structures with plural suitable directions.

This paper is organized as follows. Section 2 describes the adaptive multi-directional max-plus algebra-based morphological wavelet transform in detail. Section 3 compares the performance of the proposed transform processing with a conventional transform and other researches. Section 4 describes the expansion into multi-level AM-MMT. Finally, Section 5 concludes this paper.

\section{Adaptive Multi-Directional Max-Plus Algebra-Based Morphological Wavelet Transform}

2.1 Conventional max-plus algebra-based morphological wavelet transform

A conventional wavelet transform is known to be a signal analysis method for executing signal decomposition/reconstruction and can be classified as a linear operation [20]. The morphological wavelet transform, on the other hand, was introduced by Heijmans and Goutsias [21] as a nonlinear signal analysis method. The morphological wavelet transform combines the inherent properties of the conventional wavelet transform and mathematical morphology. However, the original morphological wavelet transform deals with the field of formulation of real numbers and several complex mathematical equations. This concept is thus difficult to implement with general-purpose processors, especially several low-power embedded mobile processors. A morphological wavelet transform, which utilizes the max-plus algebra-based concept to overcome these problems, has been proposed [22]. The Max-plus algebra-based Morphological wavelet Transform (MMT) can be defined by nonlinear operation (maximum or minimum search) and the standard sum, and it is possible to use integer representation for all variables. MMT has three novel features: no quantization error, high affinity for hardware implementation, and efficient edge image compression.

MMT decomposes the original image into several signals and then reconstructs it in accordance with the patterns of scan windows. Figure 1 shows three basic types of sampling windows (square, vertical, and horizontal) to execute the MMT processing. These sampling windows consist of four signal blocks. The variables $s_{1}, v_{1}$, and $h_{1}$ correspond to the low-frequency component. The variables $s_{4}, v_{4}$, and $h_{4}$ correspond to the high-frequency component. Other signals are high and low-frequency components.

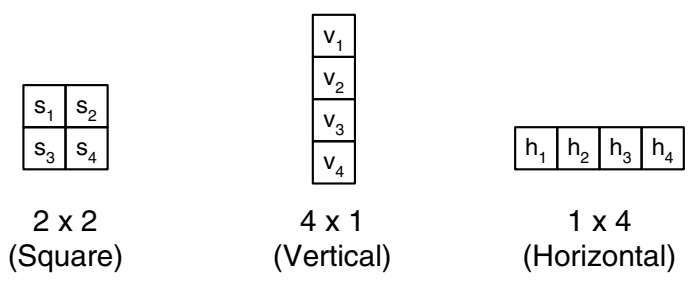

Fig. 1 Basic types of sampling windows

Figure 2 shows the decomposition/reconstruction process flow for a $2 \times 2$ pixel square sampling window. The decomposition process divides the original image $\left(2^{m} \times 2^{n}\right.$ pixels $)$ into four small squares: scaled signal, horizontal signal, vertical signal, and diagonal signal. In contrast, the original image can be reconstructed from the four pieces.

The decomposition operation in a sampling window can be represented in the following equations [23]:

$$
\begin{aligned}
& s_{1}=\min (x, y, z, w) \\
& s_{2}=y-x \\
& s_{3}=z-x \\
& s_{4}=w-x
\end{aligned}
$$

The reconstruction operation can also be represented in the following equations [23]:

$$
\begin{aligned}
x & =s_{1}+\max \left(-s_{4},-s_{2},-s_{3}, 0\right) \\
y & =s_{1}+\max \left(s_{2}-s_{3}, s_{2}-s_{4}, s_{2}, 0\right) \\
& =s_{1}+\max \left(-s_{4},-s_{2},-s_{3}, 0\right)+s_{2} \\
& =x+s_{2} \\
z & =s_{1}+\max \left(s_{3}-s_{2}, s_{3}-s_{4}, s_{3}, 0\right) \\
& =s_{1}+\max \left(-s_{4},-s_{2},-s_{3}, 0\right)+s_{3} \\
& =x+s_{3} \\
w & =s_{1}+\max \left(s_{4}-s_{2}, s_{4}-s_{3}, s_{4}, 0\right) \\
& =s_{1}+\max \left(-s_{4},-s_{2},-s_{3}, 0\right)+s_{4} \\
& =x+s_{4}
\end{aligned}
$$

Here, the four variables $x, y, z$, and $w$ correspond to the pixel values of the coordinates $(i, j),(i+1, j)$, $(i, j+1)$, and $(i+1, j+1)$ in the original image. The variables $s_{1}, s_{2}, s_{3}$, and $s_{4}$ represent decomposed images, namely, scaled, horizontal, vertical, and diagonal signals, respectively. Since the decomposition and reconstruction equations include minimum and maximum search, respectively, the max-plus algebra-based morphological wavelet transform is classified as a nonlinear operation [22].

2.2 Adaptive multi-directional max-plus algebrabased morphological wavelet transform

The Adaptive Multi-directional Max-plus algebrabased Morphological wavelet Transform (AM-MMT) 


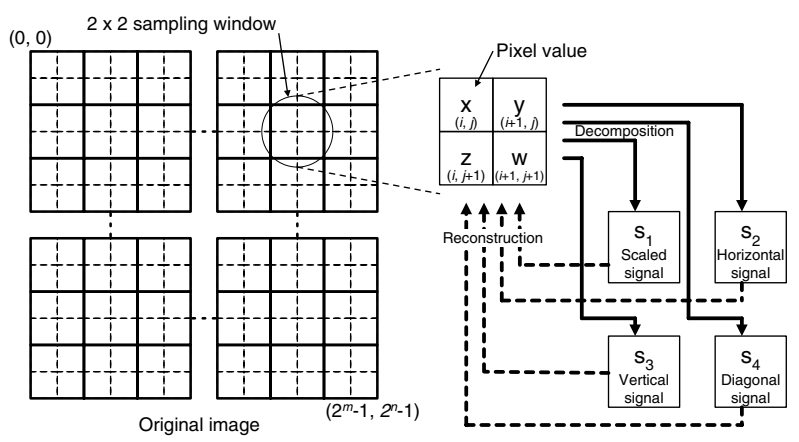

Fig. 2 Decomposition/reconstruction concept underlying max-plus algebra-based morphological wavelet transform

for realizing effective directional wavelet image processing on mobile devices is proposed and explained in this section. The proposed AM-MMT technique, which is based on the conventional MMT algorithm, can utilize several pattern sampling windows that are adaptively selected in accordance with the direction of the content in the decomposed image. Since the decomposed image is divided in a reticular pattern, the AM-MMT assumes each divided image is represented as a macro block. These macro blocks are grouped according to image structures, which are distinguished by the object-directionality of each structure in the image. Figure 3 shows the concept of the AM-MMT transform. The macro block size is defined in detail afterward (Sect. 3). The original image is decomposed by the MMT algorithm using plural sampling windows. This operation is repeatedly carried out for each sampling window. The directions of each macro block are calculated by using the results of the MMT decomposition processing. Figure 4 shows how this proposed transform adaptively selects a suitable sampling window. First, decomposed signals $s_{1}, s_{2}, s_{3}$, and $s_{4}$ are calculated by the MMT using the square sampling window. In a similar way, variables $h_{1}, h_{2}$, $h_{3}, h_{4}$ and $v_{1}, v_{2}, v_{3}, v_{4}$ are calculated in the hor- izontal and vertical sampling windows, respectively. The suitable sampling window for each macro block is selected by comparing different component signals, which are the minimum sum of absolute values, of the transform results. They are obtained by using the equations below:

$$
\underset{\left.\left|v_{3}\right|+\left|v_{4}\right|\right)}{\min \left(\sum\left|s_{2}\right|+\left|s_{3}\right|+\left|s_{4}\right|, \sum\left|h_{2}\right|+\left|h_{3}\right|+\left|h_{4}\right|, \sum\left|v_{2}\right|+\right.}
$$

Here, each summation is calculated with all the difference signals in a macro block. Selected sampling windows, which represent inherent direction, for each macro block are decided as shown in Fig. 3. Since the AM-MMT captures the directional features in the image, this transform can realize effective data compression.

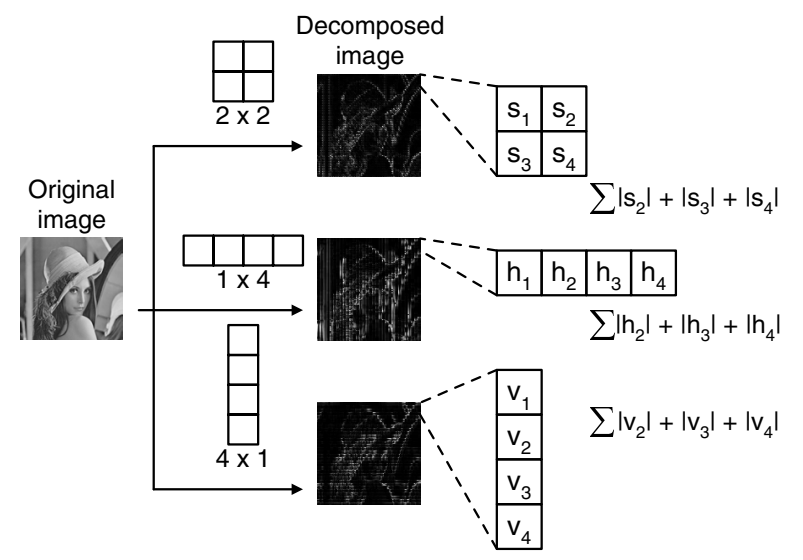

Fig. 4 How to select suitable sampling window by direction of macro block

\section{Experimental Results}

In this section, for verifying the capability of the AM-MMT, image quality and data compression are evaluated with several benchmark images to compare the AM-MMT to the conventional MMT and other researches.
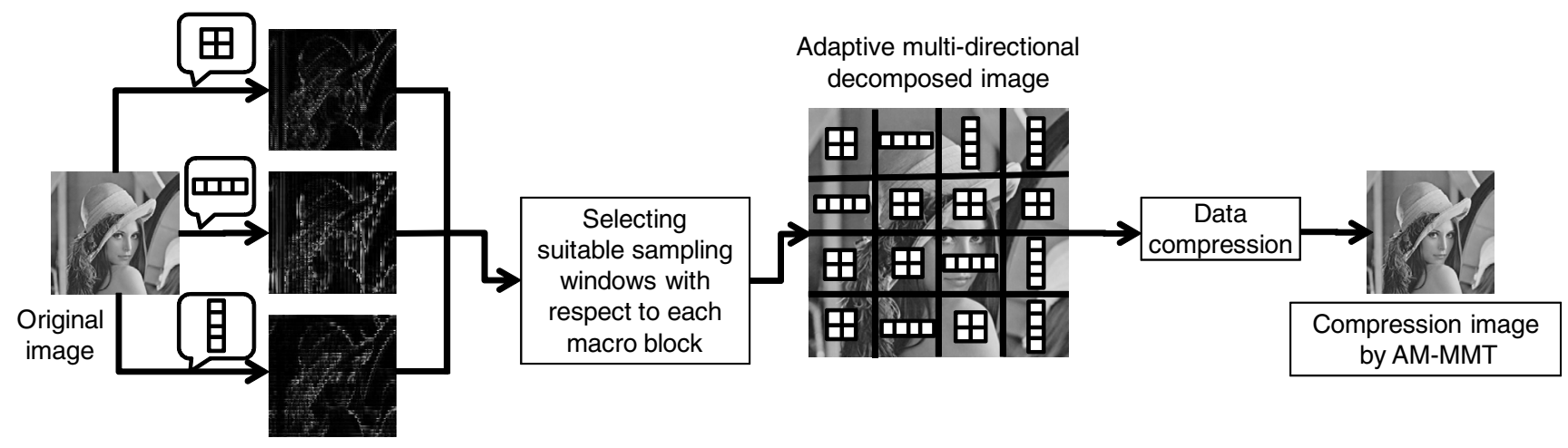

Fig. 3 Concept of AM-MMT processing 
In the experiment, the AM-MMT was been implemented with a C-language program and tested with nine standard benchmark images, such as four humans, two machines and so on. The size of these images for evaluation are $128 \times 128$ pixels. The size of the candidate sampling windows are $2 \times 2$ (square), $4 \times 1$ (vertical), and $1 \times 4$ (horizontal) pixels (Fig. 1 ). To decide on a suitable sampling window, the sum of the absolute value of different component signals is used (Sect. 2.2).

For deciding the macro block size for this experiment, decomposed results between four macro block patterns, such as $32 \times 32,16 \times 16,8 \times 8$, and $4 \times 4$ pixels, are compared (Fig. 5). Obviously, the smaller the macro block size used in this image, the more directional information can be extracted. Thus, the $4 \times$ 4 pixel macro block is applied for the decomposition and reconstruction processes below.

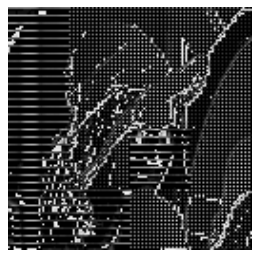

$32 \times 32$

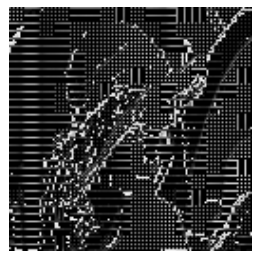

$8 \times 8$

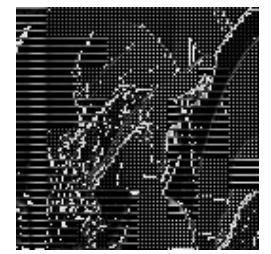

$16 \times 16$

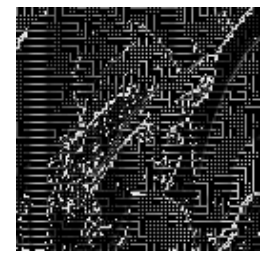

$4 \times 4$
Fig. 5 Comparison results of four macro block patterns

On the other hand, the AM-MMT needs to keep used-sampling window information for reconstruction with each change in macro block. In the case of the $128 \times 128$ pixel benchmark image with three types of sampling windows, additional sizes of used-sampling window with $32 \times 32,16 \times 16,8 \times 8$, and $4 \times 4$ pixel macro blocks are estimated to be about 0.03, 0.13, 0.50 , and $2.00 \mathrm{Kbit}$, respectively.

For the proposed wavelet transform method to be effective, compressed benchmark images of the AMMMT and those of the conventional MMT are compared. Figure 6-(a) and (b) show the original image and the AM-MMT transformed-image, respectively. Since the original image characteristically has directional information, the AM-MMT can extract the directionality of objects in the image. In this case, many $1 \times 4$ horizontal sampling windows are assigned on the left side pole. A suitable sampling window ratio is summarized in Table 1. From this table, the $1 \times 4$ sampling window is most contained and the benchmark image consists of many horizontal components. Thus, the compression results of the conventional MMT (Fig. 7-(a), (b) and (c)) and the proposed AM-MMT (Fig. 7-(d)), which can be represented by sharp-edged objects such as a woman and background, result in distinctly-different image qualities.

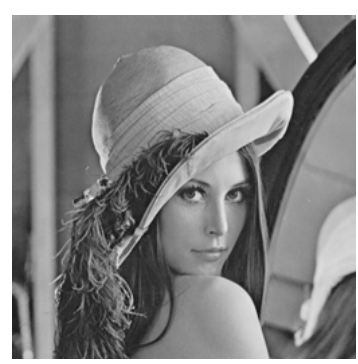

(a)

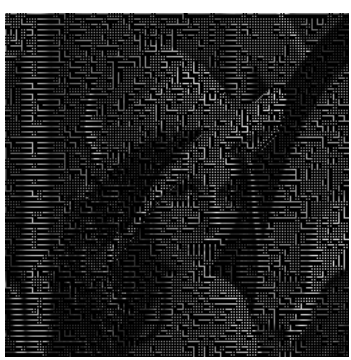

(b)
Fig. 6 Woman face benchmark: (a) Original image and (b) AM-MMT decomposed image

Table 1 Suitable sampling window percentages

\begin{tabular}{c|r|r|r}
\hline \hline $\begin{array}{c}\text { Experimental } \\
\text { sampling windows } \\
\text { [pixels] }\end{array}$ & \multicolumn{3}{|c}{ Content rate in a benchmark image [\%] } \\
\cline { 2 - 4 } & Fig. 6-(b) & Fig. 10-(b) & Fig. 13-(b) \\
\hline $2 \times 2$ (Square) & 39.9 & 39.9 & 44.4 \\
\hline $1 \times 4$ (Horizontal) & 43.8 & 21.9 & 30.3 \\
\hline $4 \times 1$ (Vertical) & 16.3 & 38.2 & 25.3 \\
\hline \hline
\end{tabular}

For evaluating the effectiveness of the proposed algorithm, the Peak Signal-to-Noise Ratio (PSNR) value, which is the most common distortion measure in the field of image quality, is calculated between the conventional MMT-compressed image and the AMMMT-processed image. The benchmark images are represented in gray-scale. Thus, the size of original image information is 128 kbit $(128$ (pixel) $\times 128$ (pixel) $\times 8$ (bit)). Figure 8 shows the estimation of compressed image data size. When the original image is compressed by AM-MMT, the bit length of the different signals in the sampling window can be decreased from 1 to 7 bit. For example, if the different signals are decreased to 4 bit, the image size becomes $80 \mathrm{kbit}$ as show in Fig. 8.

Figure 9 shows the image quality expressed in the PSNR value with 1 to 7 -bit deletion information. The number of deletion bits for the data size is indicated on the horizontal axis, and the value of PSNR is in- 


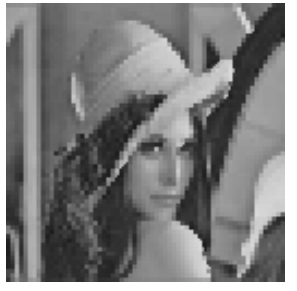

(a)

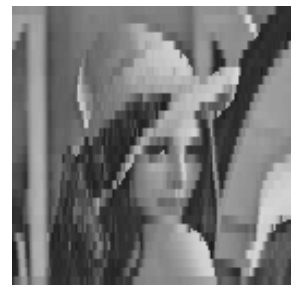

(c)

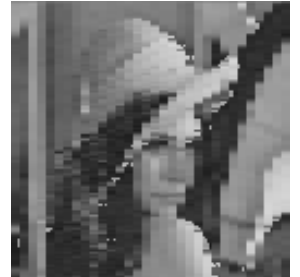

(b)

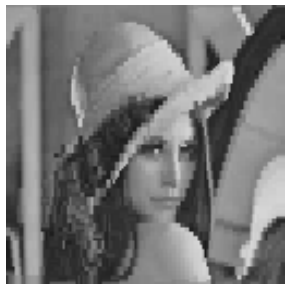

(d)
Fig. 7 Compression results of woman face benchmark: (a) conventional MMT (square sampling window), (b) conventional MMT (vertical sampling window), (c) conventional MMT (horizontal sampling window), and (d) proposed AM-MMT (adaptive sampling window)

dicated on the vertical axis. In general, a loss of image quality is hard for human eyes to notice when the overall PSNR is more than $30 \mathrm{~dB}$ [24]. The compression results of the three PSNR values of fixedsampling windows $(2 \times 2,4 \times 1$, and $1 \times 4$ pixels $)$ exceed $30 \mathrm{~dB}$ and obey these magnitude relations in accordance with the values of Table 1 . However, the actual transformed-images, which have been compressed by fixed-sampling windows, are not clear as shown in Fig. 7-(a), (b), and (c). On the other hand, the AM-MMT result (Fig. 7-(d)) clearly represents the objects'-edges. Moreover, the AM-MMT can improve on a PSNR value of about $4.60 \mathrm{~dB}$ and achieve up to about 36.59 dB even with 7-bit deletion. Thus, the re-

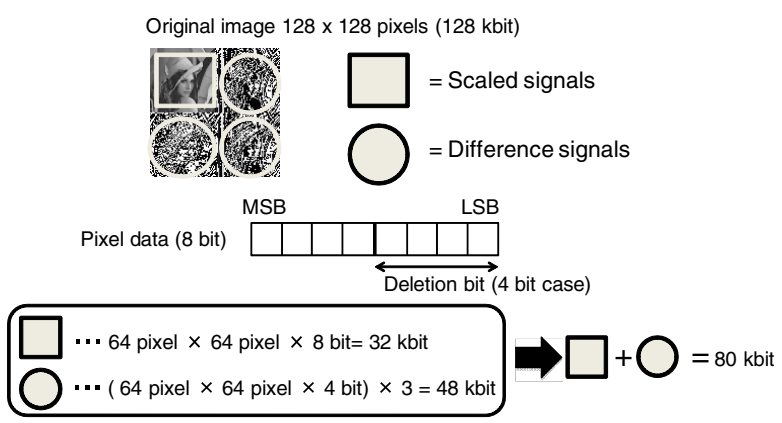

Fig. 8 Compression procedure and information size sults of the AM-MMT have enough quality for human eyes.

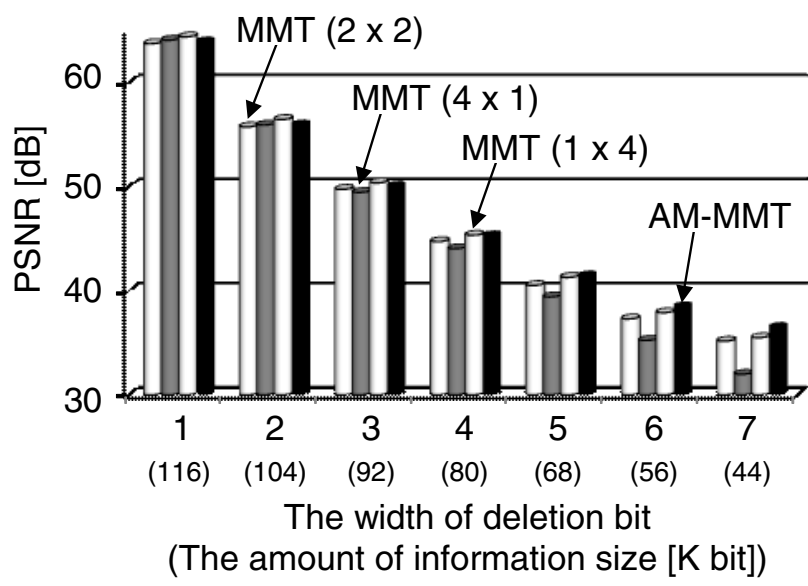

Fig. 9 Relationship between information size and PSNR by conventional MMT and proposed AM-MMT (Fig. 7)

In the airplane benchmark, which has a natural background, Fig. 10-(a) and (b), Fig. 11-(a) to (d), Fig. 12, and Table 1 show the original image, conventional MMT transformed-image, and AM-MMT transformed-image. The airplane image is occupied flattened directional structure as clouds and mountains. Thus, the square $2 \times 2$ sampling window is selected by the AM-MMT algorithm, and the vertical $4 \times 1$ sampling window is used for the airplane. Each sampling window includes about $40 \%$ in the AMMMT decomposed image, which is shown in Table 1. Thus, Fig. 11-(d) can combine the natural background of Fig. 11-(a) and the airplane of Fig. 11-(b). As a result, the image quality of the compressed image by AM-MMT can improve to about $2.85 \mathrm{~dB}$ higher than the fixed-sampling window processing with 7-bit deletion.

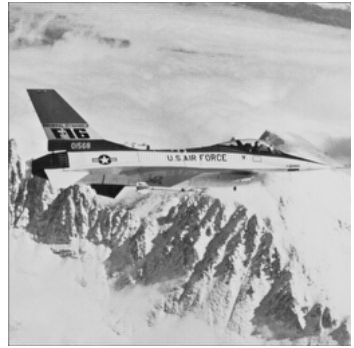

(a)

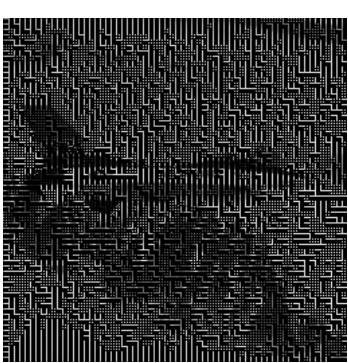

(b)
Fig. 10 Airplane benchmark: (a) Original image and (b) AM-MMT decomposed image 


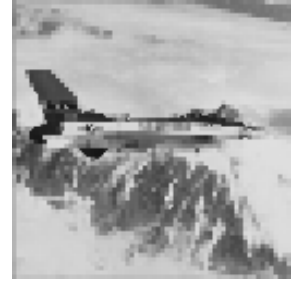

(a)

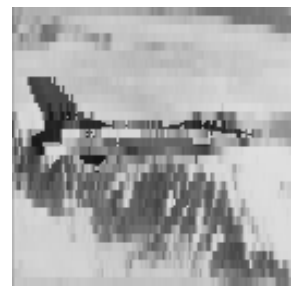

(c)

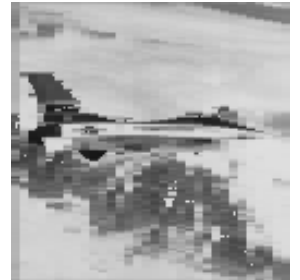

(b)

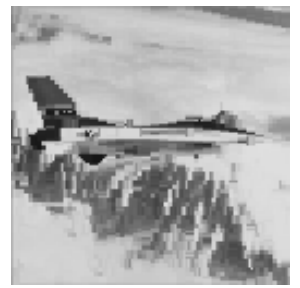

(d)
Fig. 11 Compression results of airplane benchmark: (a) conventional MMT (square sampling window), (b) conventional MMT (vertical sampling window), (c) conventional MMT (horizontal sampling window), and (d) proposed AM-MMT (adaptive sampling window)

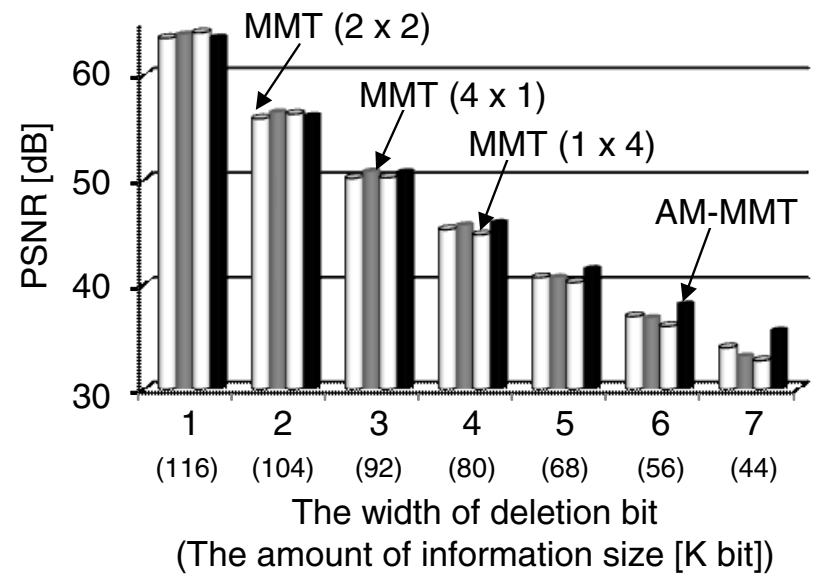

Fig. 12 Relationship between information size and PSNR by conventional MMT and proposed AM-MMT (Fig. 11)

With complex artifact benchmarks, which consist of several rods and antennae on a ship, Fig. 13-(a) and (b), Fig. 14-(a) to (d), Fig. 15, and Table 1 show effective results as well as the woman and airplane benchmark results. The AM-MMT compressed-image can sharply represent the rods on the ship (Fig. 14(d)). This image mainly combines the background of
Fig. 14-(a) and the ship of Fig. 14-(c). Furthermore, the PSNR value is up to about $3.90 \mathrm{~dB}$ higher than the PSNR values of other fixed-sampling windows.

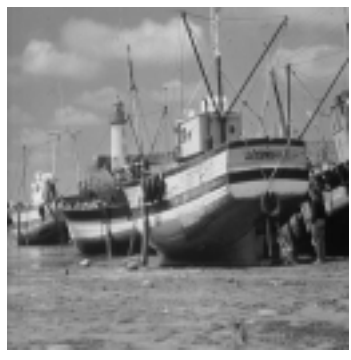

(a)

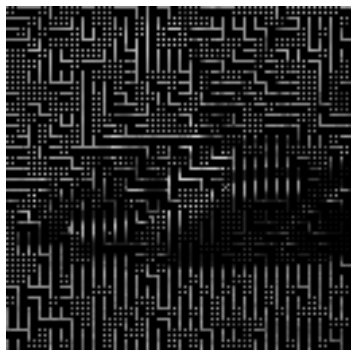

(b)
Fig. 13 Ship benchmark: (a) Original image and (b) AM-MMT decomposed image

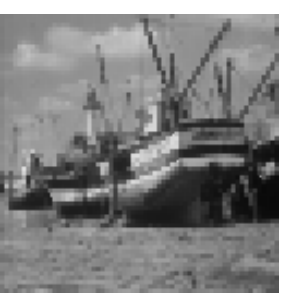

(a)

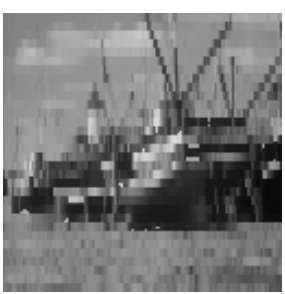

(c)

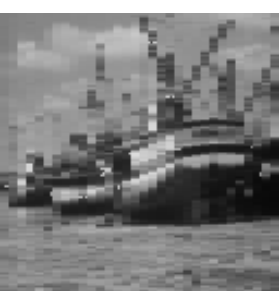

(b)

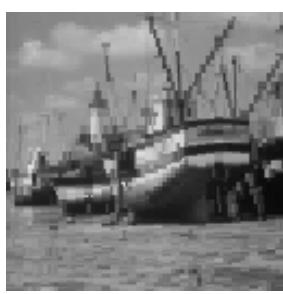

(d)
Fig. 14 Compression results of ship benchmark: (a) conventional MMT (square sampling window), (b) conventional MMT (vertical sampling window), (c) conventional MMT (horizontal sampling window), and (d) proposed AM-MMT (adaptive sampling window)

Due to limitations of space, Figure 16-(a) to (f) show six other benchmark PSNR comparison results with the conventional MMT. Almost the PSNR values of the AM-MMT are higher than that of the conventional MMT. In particular, the higher the compression degree (as shown in the deletion bit width) becomes, the lower the information size achieved when using the AM-MMT transform.

Moreover, the average size of the attached additional information for keeping used-sampling window Journal of Signal Processing, Vol. 20, No. 1, January 2016 


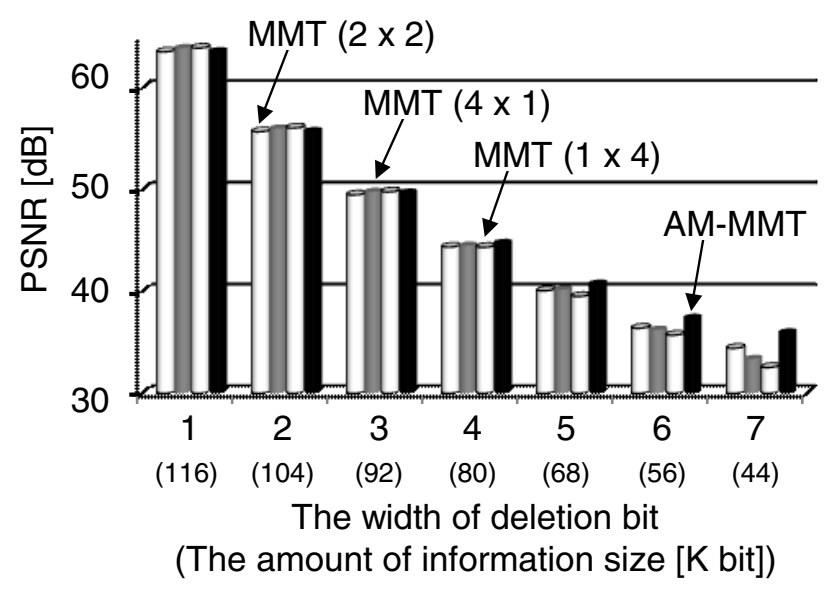

Fig. 15 Relationship between information size and PSNR when using conventional MMT and proposed AM-MMT (Fig. 14)

information is only about $2.77 \%$ of the compressed data size with 1 to 7 -bit deletion information. The effect of the additional information on the total compressed image size is only small.

Obviously, the comparison results of the conventional MMT blur the boundaries between the objects in the image. Since the AM-MMT can adaptively select suitable sampling windows, all the results have high-quality image compression and high PSNR values.

\section{Expansion into Multi-Level AM-MMT}

Several wavelet transform-based image compression methods often accelerate the compressibility ratio using multi-level decomposition and reconstruction. This operation is a well-known technique for effective performance. Thus, for further improving the image compression efficiency of the proposed method, we discuss the expansion into multi-level AM-MMT. First, this section is described as Level 2 (L2) processing below. Figure 17 shows how the L2 AM-MMT selects two sampling windows in the $4 \times 4$ pixel macro block case.

With L1 decomposition, a suitable sampling window is assigned to each macro block adaptively. On the other hand, with L2 decomposition in case of $4 \times 4$ macro block, to fit in with the shape of scaled signals, the L2 sampling windows are determined automatically. For example (gray boxes in Fig. 17), in a square sampling window of L1 AM-MMT processing, four $s_{1}$ signals are transformed into four $s_{1}^{\prime}$ signals using a square sampling window in L2 AM-MMT processing. In the same way, four $h_{1}$ signals processed by a horizontal sampling window are transformed into four $v_{1}^{\prime}$ signals, and four $v_{1}$ signals processed by a vertical sampling window are transformed into four $h_{1}^{\prime}$ signals. The above transformation can be applied to horizontal, vertical, and diagonal signals as well. Figure 18 shows pair sampling windows on L1 and L2 AM-MMT in accordance with three macro block sizes. For $4 \times 4$

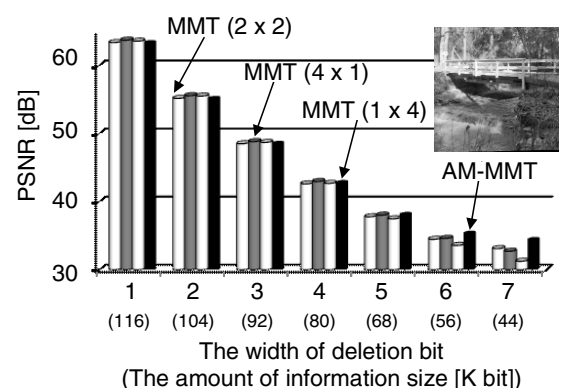

(a)

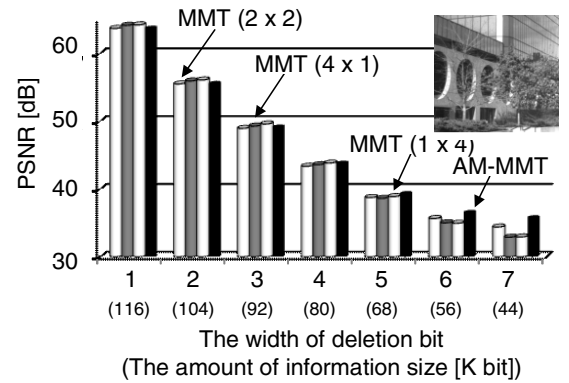

(d)

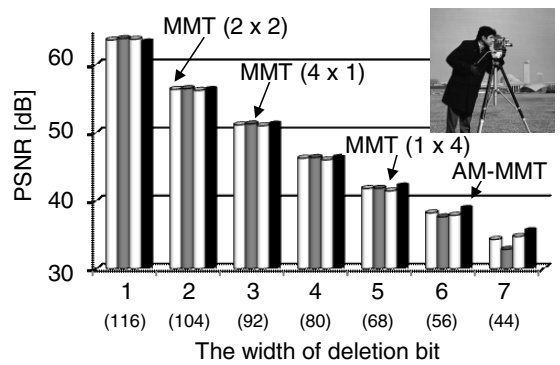

(The amount of information size [K bit]

(b)

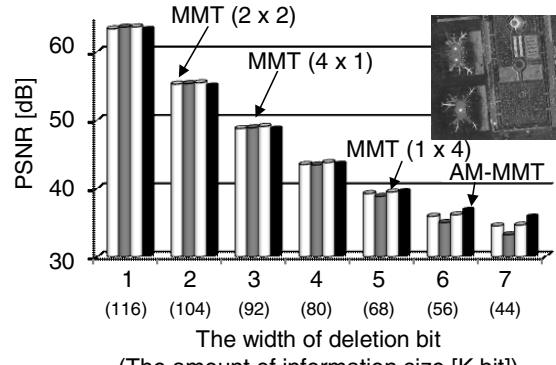

(The amount of information size [K bit])

(e)

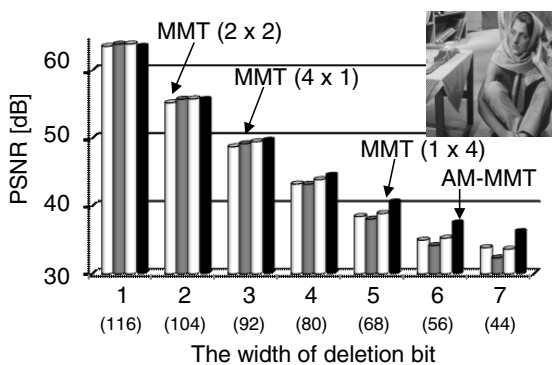

(The amount of information size [K bit])

(c)

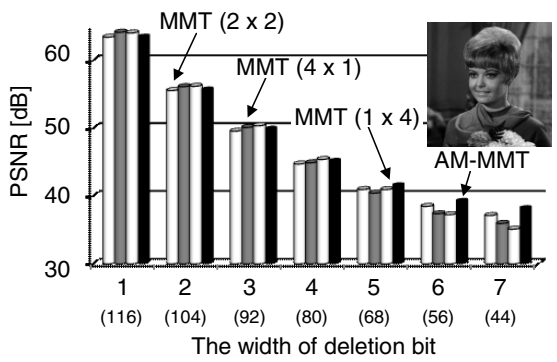

(The amount of information size [K bit])

(f)

Fig. 16 Relationship between information size and PSNR when using conventional MMT and proposed AM-MMT (six benchmark images) 
macro block, $1 \times 4$ and $4 \times 1$ sampling windows are assigned symmetrically in the L2 processing. For 8 $\times 8$ macro block, the square sampling window in the L1 processing selects all types of sampling windows in the L2 processing. The vertical and the horizontal sampling windows in the L1 processing are added the square sampling window for L2 processing. For 16 $\times 16$ macro block, all types of sampling windows are applied for the L2 processing.

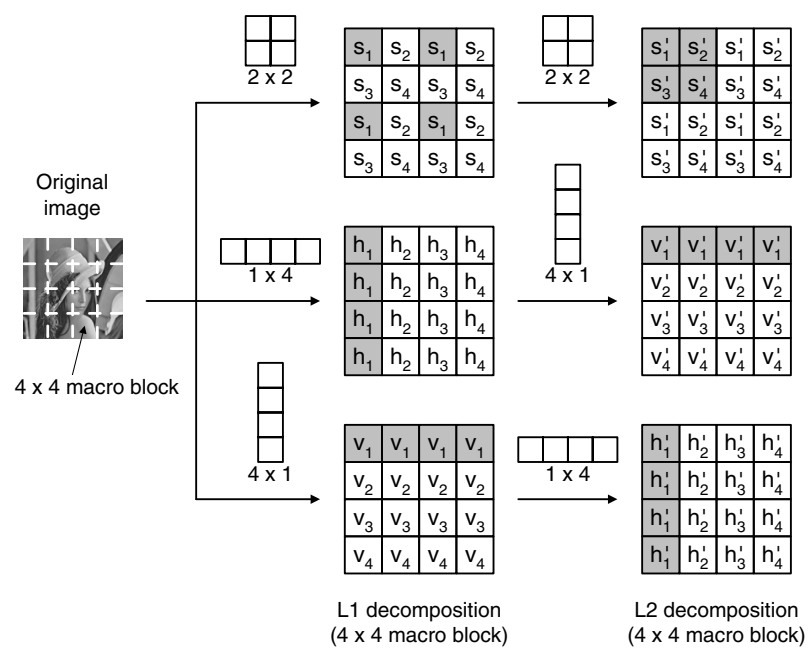

Fig. 17 Decomposition example for L2 AM-MMT on $4 \times 4$ macro block

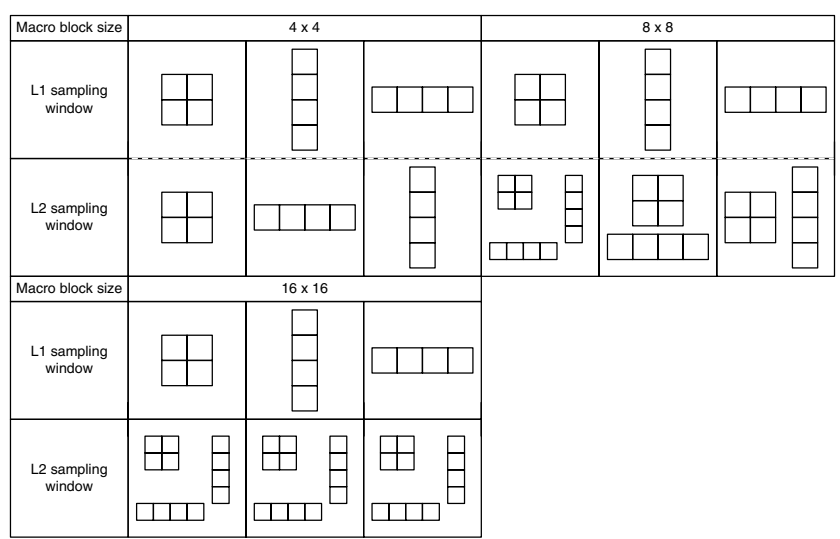

Fig. 18 Pair sampling windows for L1 and L2 MMMT in $4 \times 4,8 \times 8$ and $16 \times 16$ macro block cases

The L2 AM-MMT compression results are shown in Fig 19. For facilitating the visualization of compressed results, the size of the Lenna benchmark images and macro block for evaluation are chosen: 512 $\times 512$ and $4 \times 4$ pixels. Figure 19-(a) is an original image (2,048 Kbits). The L1 decomposition image, which is shown in Fig. 19-(b), has 7-bit deletion difference signals and a size of 704 Kbits. Thus, the L2 decomposition image will have a size of $464 \mathrm{Kbits}$ and

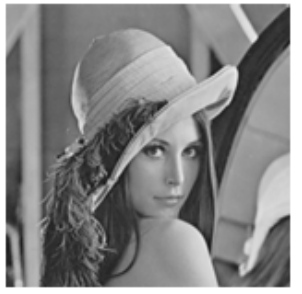

(a)

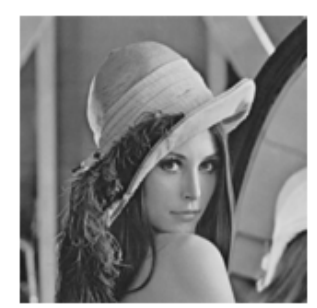

(b)

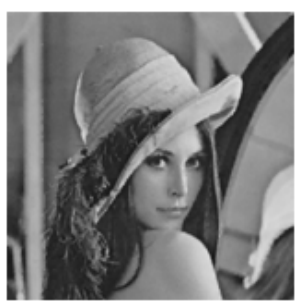

(c)

Fig. 19 Compression results of Lenna benchmark: (a) original image, (b) L1 AM-MMT compressed image, (c) L2 AM-MMT compressed image

5-bit deletion difference signals in scaled signals after L1 decomposition processing. Obviously, the L2 AMMMT can keep the image quality.

Figure 20 shows the image quality expressed in the PSNR value for the Lenna benchmark with 4 to 7 -bit and 1 to 7-bit deletion information for L1 and L2 AMMMT processing, respectively. The compressed data, which is decomposed by 7-bit deletion information for L1 and L2 AM-MMT, is up to about $82 \%$ smaller than that of the original image. Additionally, all PSNR values can be kept over $34 \mathrm{~dB}$ and have enough quality for human eyes.

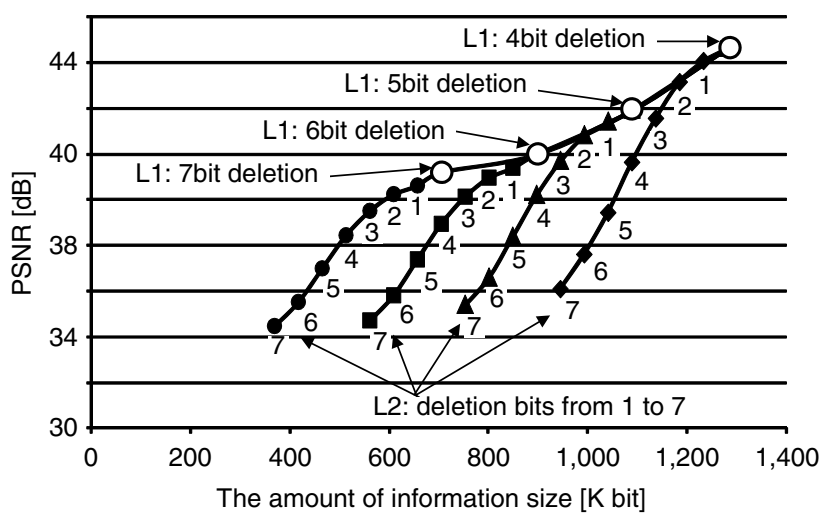

Fig. 20 Relationship between information size and PSNR when using L2 AM-MMT

Second, the generalized multi-level AM-MMT, 
which can be considered as an extension of the $2^{\alpha} \times$ $2^{\beta}$ macro block size $(\alpha, \beta=0,1,2,3, \cdots)$, is described. Here, the two variables $\alpha$ and $\beta$ correspond to the horizontal and vertical pixels of the macro block, respectively. The first-applied macro block is often square ( $\alpha=\beta, \alpha \geqq 2, \beta \geqq 2$ ) as well as the L2 AM-MMT processing. Several $2^{\alpha} \times 2^{\beta}$ macro blocks are included in the original image. Then, each macro block is applied with the square $(2 \times 2)$, vertical $(4 \times 1)$ or horizontal $(1 \times 4)$ sampling window. Three sampling windows of the generalized multi-level AM-MMT can be represented in the following equations:

\section{Square sampling window $(2 \times 2)$ $2^{\alpha} \times 2^{\beta} \longrightarrow 2^{\alpha-1} \times 2^{\beta-1}$ \\ Vertical sampling window $(4 \times 1)$ $2^{\alpha} \times 2^{\beta} \longrightarrow 2^{\alpha} \times 2^{\beta-2}$ \\ Horizontal sampling window $(1 \times 4)$ $2^{\alpha} \times 2^{\beta} \longrightarrow 2^{\alpha-2} \times 2^{\beta}$}

Generally, assigned directionality continues in the next decomposition processing after the first decomposition processing. The above sampling window operation, where the value of $\alpha$ and $\beta$ are decreasing, can be selected in accordance with the objectdirectionality and repeated until $\alpha \leqq 1$ or $\beta \leqq 1$. In this regard, the vertical and the horizontal sampling window operations are used to pair the sampling windows (Fig. 18) in $\alpha=1$ or $\beta=1$ decomposition.

Since there are three types of used sampling window (Fig. 1), two bits are needed for keeping usedsampling windows information. The additional information size, which is attached to, for example, the header area in the decrypted data, is calculated by $2^{m-\alpha} \times 2^{n-\beta} \times 2$-bit.

\section{Conclusion}

In this paper, the Adaptive Multi-directional Maxplus algebra-based Morphological wavelet Transform (AM-MMT) is proposed and evaluated. The AMMMT can extract directional structures smoothly to calculate nonlinear operation. The transformedimages of nine benchmark images show high-quality and high PSNR values. These images can be made by combining the high quality parts of each sampling window. Thus, all PSNR values of the AM-MMT are higher than those of the conventional MMT with increasing deletion bit width. Furthermore, the expansion into the L2 AM-MMT compressed-image achieves an image that is up to about $82 \%$ smaller than the original image. Therefore, the proposed AM-MMT transform method can achieve efficient data transformation for several multimedia applications.

\section{Acknowledgments}

Part of this work has been supported by a Grantin-Aid for Young Scientists (B) (No. 24710190), Ministry of Education, Culture, Sports, Science and Technology, Japanese Government.

\section{References}

[1] T. Tohne, H. Koda and H. Tanaka: Directional filter constructed via discrete cosine transform and its application to image coding, IEICE Trans. Inf. \& Syst., Vol. J76-D-II, No. 12, pp. 2633-2637, 1993.

[2] M. Naemura, A. Fukuda, Y. Mizutani, Y. Izumi, K. Yamaguchi and Y. Ninomiya: Detection of image orientation with mathematical morphology and orientation-adaptive image processing for antialiasing, IEICE Trans. Inf. \& Syst., Vol. J80-D-II, No. 10, pp. 2733-2743, 1997.

[3] S. Doi, E. Ueda and S. Doi: A directional morphological operation and its application to immunological image processing, Proc. IEEE Int. Conf. on Acoustic, Speech and Signal Processing, Vol. 4, pp. 3153-3156, 1997.

[4] P. Soille and H. Talbot: Directional morphological filtering, IEEE Trans. Pattern Analysis and Machine Intelligence, Vol. 23, No. 11, pp. 1313-1329, 2001.

[5] G. Milici, G. Raia, S. Vitabile and F. Sorbello: Fingerprint image enhancement using directional morphological filter, Proc. IEEE Int. Conf. on EUROCON, pp. 967-970, 2005.

[6] H. Sakano and T. Yamada: Why directional feature work well?, IEICE Technical Report, Vol. PRMU2008-146, pp. 141-24, 2008

[7] M. Ikehara: Front of multi-directional wavelets and multidimensional filter banks: directional adaptive image coding, J. IEICE, Vol. 92, No. 8, pp. 706-712, 2009.

[8] S. Valero, J. Chanussot, J. A. Benediktsson, H. Talbot and B. Waske: Directional mathematical morphology for the detection of the road network in very high resolution remote sensing images, Proc. IEEE Int. Conf. on Image Processing, pp. 3725-3728, 2009.

[9] J. Xu, B. Zeng and F. Wu: An overview of directional transforms in image coding, Proc. IEEE Int. Conf. on Circuits and Systems, pp. 3036-3039, 2010.

[10] J. Xu and F. Wu: Subsampling-adaptive directional wavelet transform for image coding, IEEE Data Compression Conference, pp. 89-98, 2010.

[11] S. Yoshioka and T. Tanaka: Orientation adaptive coding of images exploiting JPEG codec, IEICE General Conference, No. A-4-17, pp. 95, 2011.

[12] D. Jayachandra and A. Makur: Directionlets using inphase lifting for image representation, IEEE Trans. Image Processing, Vol. 23, No. 1, pp. 240-249, 2014.

[13] T. Kumaki, M. Osawa, S. Itaya, T. Ogura and T. Fujino: Decomposition/Reconstruction acceleration of maxplus algebra-based morphological wavelet transform with massive-parallel SIMD matrix mobile processor, Journal of Signal Processing, Vol. 15, No. 6, pp. 425-434, Nov., 2011.

[14] T. Kumaki, Y. Murakami, S. Itaya, K. Nakao, T. Ogura and T. Fujino: Max-plus algebra-based morphological wavelet transform watermarking for highly-parallel pro- 
cessing with mobile embedded processor, Journal of Signal Processing, Vol. 16, No. 6, pp. 547-556, Nov., 2012.

[15] M. Nawata, S. Shirai, M. Nakai, T. Kumaki, T. Fujita and T. Ogura: Compression efficiency study of directional morphological wavelet transform, Proceedings of RISP International Workshop on Nonlinear Circuits, Communications and Signal Processing, pp. 100-102, 2012.

[16] Y. Tanito, T. Tokunaga, M. Nawata, T. Kumaki, T. Fujita and T. Ogura: Adaptive multi-directional morphological wavelet transform and it's compression efficiency, Proceedings of RISP International Workshop on Nonlinear Circuits, Communications and Signal Processing, pp. 305-308, 2013.

[17] T. Kumaki, T. Fujita, M. Nakanishi and T. Ogura: Morphological pattern spectrum and block cipher processing based image-manipulation detection, IEICE Trans. Nonlinear Theory and Its Applications, Vol. 4, No. 4, pp. 400-418, 2013.

[18] T. Tokunaga, Y. Tanito, T. Kumaki, T. Fujita and T. Ogura: L2 decomposition of adaptive directional morphological wavelet transform, Proceedings of RISP International Workshop on Nonlinear Circuits, Communications and Signal Processing, pp. 137-140, 2014.

[19] T. Kumaki, K. Nakao, K. Hozumi, T. Ogura and T. Fujino: Development of compression tolerable and highly implementable watermarking method for mobile devices, IEICE Trans. Inf. \& Syst., Vol. E97-D, No. 3, pp. 593-596, 2014.

[20] H. Nobuhara: Non-linear wavelets by various sampling windows, IEICE Technical Report, Vol. SIS2006-4, pp. 19-23, 2006.

[21] H. J. A. M. Heijmans and J. Goutsias: Nonlinear multiresolution signal decomposition schemes-part II: Morphological wavelets, IEEE Trans. Image Processing, Vol. 9, No. 11, pp. 1897-1913, 2000.

[22] H. Nobuhara, K. Hirota and B. Bede: Max-plus algebra based wavelet transform and its application to video compression/reconstruction, Proc. IEEE International Conference on Image Processing, pp. 1785-1788, 2006.

[23] A. Asano, C. M. Asano, Y. Kimori, M. Muneyasu, H. Nobuhara and M. Fujino: Nonlinear image and signal processing - basics and applications of mathematical morphology, Maruzen, pp. 69-78, 2010.

[24] http://supercompression.com/e/motionpicture.html

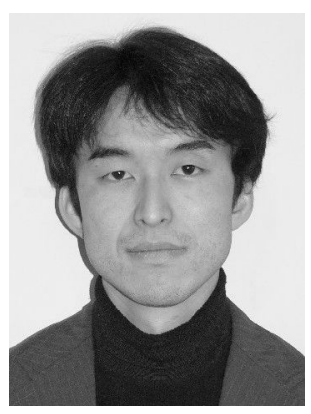

Takeshi Kumaki received his B.S. degree from the Department of Mathmatics, Faculty of Science and completed the first half of the M.E. program in information mathematics from National Defence Academy, Kanagawa, Japan in 1998 and 2003, respectively, and Ph.D., degree in electric engineering from Hiroshima University, Hiroshima, Japan in 2006. From 2003 to 2004, he was affiliated with the Japan Air SelfDefence Force Electric Experimentation Group. From 2005 to 2009, he joined the Research Center for Nanodevices and Systems (RCNS) and the Research Institute for Nanodevice and Bio
Systems (RNBS), Hiroshima University, Japan, where he has engaged in the system design and architecture research. From 2010 to 2012, he became an Assistant Professor in the Department of VLSI System Design, Ritsumeikan University. Since 2013, he has been a Lecturer in the Department of Electronic and Computer Engineering, Ritsumeikan University. He is interested in content addressable memory, SIMD processing architecture and these applications. Dr. Kumaki is a senior member of the Institute of Electrical and Electronics Engineers (IEEE), the Institute of Electronics, Information and Communication Engineers of Japan (IEICE) and the Research Institute of Signal Processing (RISP).

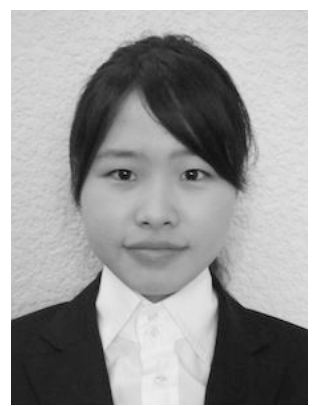

Yuri Tanito received her B.S. and M.E. degrees in the Department of VLSI System Design from Ritsumeikan University in 2012 and 2014, respectively. She is interested in morphological image processing.

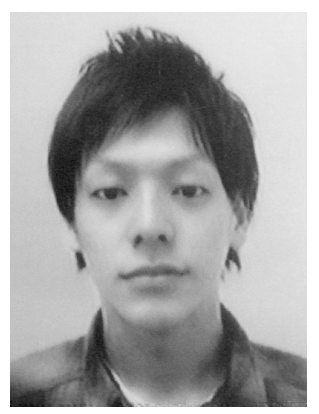

Tatsuki Tokunaga received his B.S. and M.E. degrees in the Department of VLSI System Design from Ritsumeikan University in 2013 and 2015, respectively. Now, he works in a car parts manufacturer and aims to make high quality and safety car parts.

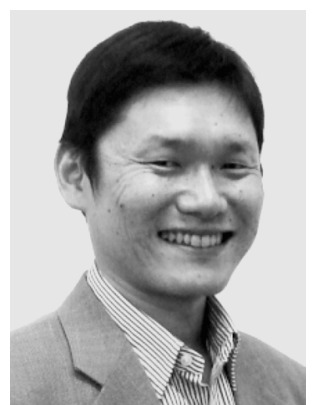

Tomohiro Fujita received his B.E., M.E., and Ph.D. degrees from Kyoto University, Kyoto, Japan, in 1991 1993, and 2001 respectively. Form 1993 to 1997 he worked at Mitsubishi Electric Corporation. Since 2001 he has been a Lecturer (20012003), an Associate Professor (20042010) in the Department of VLSI System Design, Ritsumeikan University. Since 2011, he has been a Professor in the Department of Electronic and Computer Engineering, Ritsumeikan University. His research interests include computer-aided design of analog LSIs and cellular neural network. He is a member of the IEEE, IEICE and RISP. 


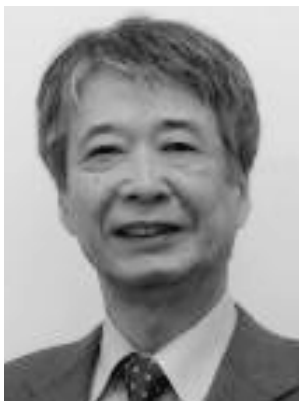

Takeshi Ogura received his B.S., M.S., and Ph.D. degrees in electrical engineering from Osaka University, Osaka, Japan, in 1976, 1978, and 1991, respectively. In 1978, he joined Nippon Telegraph and Telephone Corp.(NTT). In NTT, he engaged in the research and development of CAM LSIs and image encoding LSIs, and their applications. Since 2004 he has been a Professor in the Department of VLSI System Design, Ritsumeikan University, Shiga, Japan. Dr. Ogura is a member of the IEEE, IEICE, and IPSJ.

(Received April 8, 2015; revised June 23, 2015) 